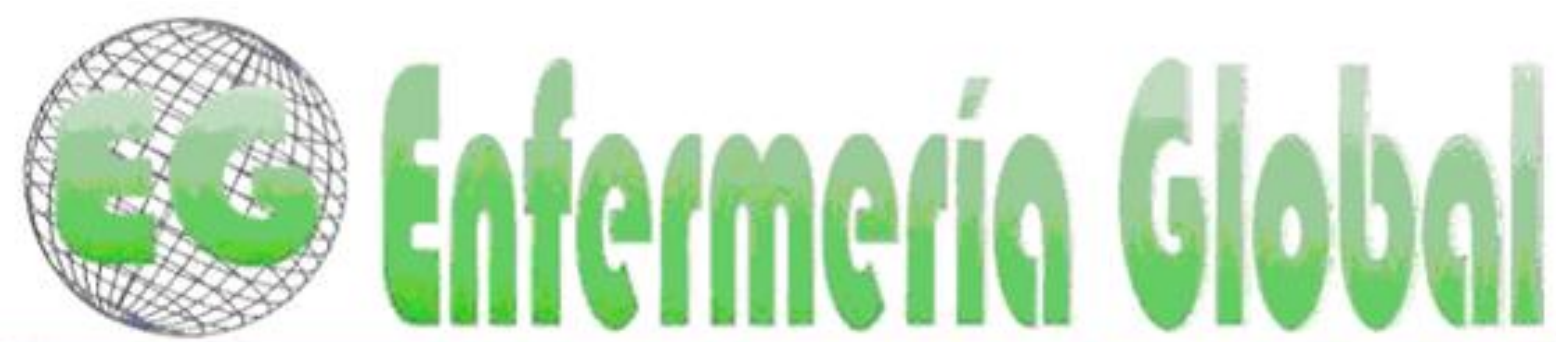

ISSN 1695-6141

$N^{\circ} 44$

Revista electrónica trimestral de Enfermería

www.um.es/egloball

Octubre 2016

DOCENGIA - INVESTIGACIÓN

\title{
Situación actual de la especialidad de Enfermería Familiar y Comunitaria en España
}

State speciality Family and Community Nursing in Spain

\section{${ }^{*}$ Represas-Carrera, Francisco Jesús ${ }^{*}$ Comesaña-Diego, Lorena ${ }^{* *}$ Carrera- García, Álvaro ${ }^{* * *}$ Vidal Fernández-Fernández, Manuel ${ }^{* * * *}$ Rodríguez- Iglesias, Francisco Javier ${ }^{* * * *}$ Calvo-Pérez, Ana Isabel ${ }^{* * * * * *}$ Díaz-Hoyos, Rita}

*Enfermero/a Especialista en Enfermería Familiar y Comunitaria. Asociación Gallega de Enfermería Familiar y Comunitaria (AGEFEC). E-mail: franciscorepresascarrera@gmail.com **Enfermero Interno Residente de Enfermería Familiar y Comunitaria. (AGEFEC). "***Enfermero de Atención Primaria en el Centro de Salud de Sigüeiro (A Coruña). (AGEFEC) ${ }^{* * * * E n f e r m e r o ~ d e ~ A t e n c i o ́ n ~ P r i m a r i a ~}$ en el Centro de Salud de Órdenes (A Coruña). (AGEFEC) ${ }^{* * * * *}$ Enfermera del Servicio de Integración Asistencial de la Dirección General de Asistencia Sanitaria (Servicio Gallego de Salud). (AGEFEC)

******Enfermera de Atención Primaria en el Centro de Salud de Malpica (A Coruña). (AGEFEC)

España.

Palabras clave: Enfermería; Enfermería en Salud Comunitaria; Especialidades de Enfermería; Sociedades Científicas

Keywords: Nursing; Community Health Nursing; Nursing Specialties; Societies Scientific..

\section{RESUMEN}

Objetivo: Conocer la realidad sobre la aplicación del programa formativo en cada una de las Unidades Docentes Multiprofesionales de Atención Familiar y Comunitaria, y también describir la situación actual y perspectivas laborales de los actuales especialistas de Enfermería Familiar y Comunitaria.

Método: Estudio descriptivo de corte transversal realizado durante los meses de Septiembre y Octubre del 2014 a los profesionales residentes y especialistas de Enfermería Familiar y Comunitaria de toda España. Para obtener los datos se facilitó de forma online un cuestionario autoadministrado de carácter voluntario y anónimo, el cual fue elaborado por un grupo de expertos en la especialidad de Enfermería Familiar y Comunitaria pertenecientes a la Asociación Gallega de Enfermería Familiar y Comunitaria de Galicia (AGEFEC).

Resultados: Se estudió una muestra de 342 profesionales. El $69 \%$ eran residentes y el $31 \%$ 
especialistas en Enfermería Familiar y Comunitaria. El 54\% de los especialistas y el $56 \%$ de los residentes indican un déficit en la adquisición de competencias recogidas en el programa formativo de su especialidad. El $46 \%$ de los especialistas está trabajando actualmente en el ámbito de la Atención Primaria.

Conclusiones: Resulta de vital importancia ofrecer una formación especializada en Enfermería Familiar y Comunitaria lo más homogenizada posible, sirviendo como un instrumento necesario para garantizar la calidad de la formación en todo el sistema sanitario.

\section{ABSTRACT}

Objective: Knowing the reality on the implementation of the training program in each of the teaching units Multiprofessional Family Care and Community, and also to describe the current situation and perspectives of current labor specialists of Family and Community Nursing.

Method: Descriptive cross-sectional study conducted during the months of September and October 2014 resident's professionals and specialists of Family and Community Nursing throughout Spain. For data was provided so online a self-administered questionnaire voluntary and anonymous, which was developed by a group of experts in the specialty of Family and Community Nursing belonging to the Galician Association of Family and Community Nursing Galicia (AGEFEC).

Results: A sample of 342 professionals was studied. $69 \%$ were residents and $31 \%$ specialists in Family and Community Nursing. $54 \%$ of specialists and $56 \%$ of residents indicate a deficit in the acquisition of skills included in the training program for their specialty. $46 \%$ of specialists are currently working in the field of primary care.

Conclusions: It is important to provide a specialized training Family and Community Nursing homogenized as possible, serving as a necessary step to ensure the quality of training throughout the health system instrument.

\section{INTRODUCCIÓN}

La sociedad actual presenta unas demandas en salud derivadas de circunstancias concretas: factores demográficos y económicos, patologías prevalentes, tecnologías de la información, movimientos poblacionales, hábitos de salud, derechos del ciudadano, etc ${ }^{(1-4)}$. La especialidad de Enfermería Familiar y Comunitaria, a través de cuidados de calidad asegura un ejercicio profesional basado en los valores profesionales que la legitiman ante la sociedad, dando respuesta a las demandas de salud que se deriven de los factores antes citados ${ }^{(5-7)}$.

El derogado Real Decreto 450/2005 sobre especialidades de enfermería posibilitó el desarrollo del programa formativo de la especialidad de Enfermería Familiar y Comunitaria ${ }^{(8)}$. En la actualidad esta especialidad se regula, junto al resto de especialidades enfermeras, por el Real Decreto 639/2014 ${ }^{(9)}$, lo que supone un paso importante en el modelo de desarrollo de la profesión enfermera diseñado por la Ley $44 / 2003$, de ordenación de las profesiones sanitarias ${ }^{(10)}$.

Desde la Asociación Gallega de Enfermería Familiar y Comunitaria (AGEFEC) y junto a todas las Sociedades Científicas de Enfermería Familiar y Comunitaria de Atención Primaria que integran la federación de FAECAP, hacemos una apuesta clara e indiscutible por la especialidad de Enfermería Familiar y Comunitaria en todos los aspectos ${ }^{(6,11-13)}$. Sin duda, la incorporación de los enfermeros especialistas en formación supone un reto y un estímulo para los tutores, pero también un enriquecimiento personal y profesional para ambos y una mejora en la calidad y cantidad de cartera de servicios que se oferta a la ciudadanía ${ }^{(11,14,15)}$. 
Para la mejora continua de la docencia y de las condiciones de formación de los Enfermeros Internos Residentes (EIRs) de la especialidad de Enfermería Familiar y Comunitaria, es de vital importancia ofrecer una formación con un alto estándar de calidad, para tratar así de alcanzar todas las competencias descritas en el programa formativo de la especialidad ${ }^{(5,16,17)}$; consiguiendo formar a los profesionales que serán la guía de la Enfermería de Atención Primaria en los próximos años ${ }^{(2,6,7,18)}$.

Conocer cómo se está desarrollando el programa formativo de la especialidad de Enfermería Familiar y Comunitaria en todo el territorio Español servirá para ayudar a mejorar la formación en los próximos años. Por ello el objetivo de este estudio es conocer la realidad sobre la aplicación del programa formativo en cada una de las Unidades Docentes Multiprofesionales de Atención Familiar y Comunitaria, y describir la situación actual y las perspectivas laborales de los especialistas de Enfermería Familiar y Comunitaria.

\section{MATERIAL Y MÉTODOS}

-Diseño: Estudio observacional de tipo descriptivo transversal.

-Ámbito y período de estudio: Este estudio fue realizado a los enfermeros especialistas y residentes de Enfermería Familiar y Comunitaria de todo España durante los meses de Septiembre y Octubre del 2014.

-Sujetos: Los sujetos seleccionados fueron enfermeros especialistas y residentes en Enfermería Familiar y Comunitaria que durante el período de estudio estuvieron trabajando en España. La población diana comprendía a 809 personas de las que se obtuvieron datos de 342 mediante un muestreo consecutivo durante los dos meses que duró la recogida de datos, siendo esta una muestra representativa de la población a estudio atendiendo a una estimación de error del 5\%, un intervalo de confianza del $95 \%$ y una distribución del $50 \%$.

-Variables estudiadas: Se estudiaron variables socio-demográficas (edad, año de promoción, sexo, categoría profesional, año de residencia) y las variables de instrumento incluidas en el anexo I.

-Recogida de datos: Durante el período de estudio se facilitó de forma online un cuestionario autoadministrado de carácter voluntario y anónimo a todos los residentes y especialistas de Enfermería Familiar y Comunitaria existentes en el estado Español hasta la fecha de Septiembre del 2014. Este cuestionario fue elaborado por un grupo de expertos en la especialidad de Enfermería Familiar y Comunitaria pertenecientes a la Asociación Gallega de Enfermería Familiar y Comunitaria de Galicia (AGEFEC) y pilotado previamente en un grupo de sujetos con las mismas características que la población estudiada. Se adjunta el cuestionario como anexo I.

-Análisis de los datos: Los datos fueron recogidos y procesados en Microsoft Excel 2012, para realizar el análisis descriptivo se utilizó el SPSS versión 21. Las variables cualitativas se reportaron como frecuencias absolutas y relativas, las variables cuantitativas se presentaron como media y desviación estándar.

-Consideraciones éticas: En todos los casos los profesionales de enfermería fueron informados acerca de los objetivos del estudio, de cómo se pretendía llevarlo a cabo y de la participación que precisábamos por su parte. Todos los profesionales dieron su 
consentimiento para participar en él. Son tenidas presentes las recomendaciones establecidas por la Ley Orgánica de Protección de Datos 15/1999 y la Ley de Protección de datos de carácter personal.

\section{RESULTADOS}

Los datos socio-demográficos de la población estudiada se representan en la tabla 1. A la preferencia por la que tuvieron que escoger, una vez superado el examen de acceso, la especialidad de Enfermería Familiar y Comunitaria el $70 \%$ indica que fue su primera opción, un $23 \%$ su segunda opción, un $6 \%$ su tercera opción y un $1 \%$ su cuarta opción. En el supuesto caso de tener que volver a escoger una especialidad enfermera, el $90 \%$ indica que la especialidad de Enfermería Familiar y Comunitaria sería su primera opción, un $7 \%$ su segunda opción, un $2 \%$ su tercera opción y un $1 \%$ su cuarta opción.

En la tabla 2 se indican los ámbitos competenciales en donde el $54 \%$ de los especialistas tuvieron mayor dificultad para adquirir las competencias reflejadas en el programa formativo.

La organización de la parte teórica de la formación en las Unidades Docentes Multiprofesionales de Atención Familiar y Comunitaria se realiza en un 65\% exclusivamente mediante cursos de formación y en un 35\% a través de clases teóricas junto a cursos de formación.

En el $52 \%$ de los casos la presidencia de la subcomisión de docencia de las Unidades Docentes está ocupada por un profesional de enfermería y en un $32 \%$ por un profesional médico mientras que el $16 \%$ de los encuestados desconoce la categoría profesional de este cargo en su Unidad Docente.

El apoyo a la investigación que ofrecen las diferentes Unidades Docentes es valorado cuantitativamente mediante una escala de Likert del uno al cinco, significando el uno "nada satisfecho" y el cinco "totalmente satisfecho". Los sujetos puntuaron este ítem con un media de 3,14 . Refiriendo en un $51 \%$ de los casos la exigencia de realizar un trabajo de investigación como requisito para optar al título de especialista en Enfermería Familiar y Comunitaria, y en un $49 \%$ la exigencia de realizar un proyecto de investigación. Permitiéndose de media 3 investigadores por trabajo o proyecto de investigación.

En los aspectos relacionados con la figura del tutor el $79 \%$ valora su calidad asistencial de forma excelente o muy buena, el $17 \%$ lo valora con una calidad asistencial buena y un $4 \%$ como regular o pobre. En relación a su calidad docente, el $67 \%$ la valora como excelente o muy buena, el $19 \%$ como buena y un $14 \%$ como regular o pobre.

El $61 \%$ de los encuestados responde afirmativamente a la pregunta realizada sobre si la carga asistencial en la consulta de su tutor en Atención Primaria facilita docencia.

El registro de la actividad formativa se realiza en un $54 \%$ mediante un sistema de portafolios y en un $42 \%$ mediante el libro del residente, mientras que el $4 \%$ de los encuestados refiere no registrar su actividad formativa. 
Las Sociedades Científicas de Enfermería Familiar y Comunitaria son conocidas por el $92 \%$ de los encuestados, perteneciendo a ellas el $53 \%$ de estos. Un $59 \%$ considera que estas organizaciones están velando por el desarrollo de todos los aspectos relacionados con la especialidad de Enfermería Familiar y Comunitaria, mientras que el $20 \%$ no comparte esta afirmación y el $21 \%$ lo desconoce.

Al cuantificar mediante una escala de Likert del uno al cinco, significando el uno "nada" y el cinco "mucho", el esfuerzo que realizan las Sociedades Científicas de Enfermería Familiar y Comunitaria para lograr la inserción laboral de los especialistas en el ámbito de la Atención Primaria. Los participantes en este estudio puntuaron este ítem con un media de 3,10, siendo la Asociación Gallega de Enfermería Familiar y Comunitaria (AGEFEC) la Sociedad Científica mejor puntuada con una nota media de 4,20 .

En relación a la situación laboral de los especialistas en Enfermería Familiar y Comunitaria, el $46 \%$ está trabajando actualmente en el ámbito de la Atención Primaria. De los enfermeros especialistas que se encuentran trabajando en Atención Primaria el $75 \%$ lo hacen mediante un contrato eventual, el $21 \%$ mediante una interinidad y un $4 \%$ por vinculación fija. El $65 \%$ de los especialistas explica que el hecho de poseer este título no le ha facilitado su inserción laboral en el ámbito de la Atención Primaria, y el $48 \%$ de los encuestados cree que la situación actual de los especialistas cambiará en los próximos 2 años.

El $75 \%$ de los profesionales de enfermería afirma que los dos años de formación no se están valorando en bolsas de contratación de los servicios de salud de las distintas Comunidades Autónomas del estado Español.

Los especialistas y residentes de Enfermería Familiar y Comunitaria consideran prioritario conseguir la creación de su categoría profesional en los diferentes servicios de salud (48\%), en segundo lugar conseguir la creación de bolsas de trabajo que favorezcan el acceso de los especialistas a la Atención Primaria (42\%) y por último sitúan la celebración de la prueba de acceso para optar al título de la especialidad por la vía extraordinaria (10\%).

Preguntándoles a los encuestados sobre si han tenido contacto con las diferentes Organizaciones Sindicales para exponerles la actual problemática que existe con la situación laboral, el 55\% afirma que sí han tenido contacto y el restante $45 \%$ todavía no.

Finalmente en la tabla 4 los residentes y especialistas nos muestran su opinión sobre qué puede aportar un especialista en Enfermería Familiar y Comunitaria a la Atención Primaria. Está tabla fue elaborada una vez se eliminaron las contestaciones duplicadas.

\section{DISCUSIÓN}

Después de que en el año 2010 se aprobara el programa formativo de la especialidad de Enfermería Familiar y Comunitaria ${ }^{(2)}$ se ofertaron las primeras 132 plazas de formación sanitaria especializada ${ }^{(8)}$. El actual Sistema Nacional de Salud ya cuenta con profesionales de Enfermería especialistas en Enfermería Familiar y Comunitaria, tal y como se refleja en los resultados de nuestro estudio. $Y$ también que el número de profesionales de enfermería que se están formando en esta especialidad va creciendo 
año tras año, justificando así el aumento de la población estudiada en cada promoción formativa. Aunque a pesar de que exista un aumento en el número de plazas varios estudios denuncian que esta oferta sigue siendo insuficiente ${ }^{(1,7,11)}$.

La adquisición de las competencias es situada por otros autores como un elemento fundamental para la constitución de una profesión desarrollada y madura, autónoma y con capacidad de dar servicio útil a la sociedad ${ }^{(6,7,13)}$; sin embargo, el $33 \%$ de los especialistas y el $44 \%$ de los residentes considera que no han adquirido todas las competencias relacionadas con la investigación que aparecen recogidas en el programa formativo. La correcta adquisición de las competencias en investigación es cuestionada por diversos estudios en donde se relatan dificultades generadas por la incorporación de tutores clínicos enfermeros que se inician en esta actividad docente lo que parece que no consigue garantizar la adquisición de estas competencias por parte de las enfermeras especialistas en formación ${ }^{(14-16)}$.

El aprendizaje en investigación de una enfermera especialista se ve reforzado por la exigencia, durante el período de residencia, del diseño tutorizado de un proyecto de investigación ${ }^{(2)}$ como así se describe en los resultados de este estudio.

No queda excluida de la formación teórica de los especialistas la realización de talleres o seminarios formativos. Aquí también existe una gran variabilidad formativa dependiendo de cada Unidad Docente en cuanto a la metodología de su formación teórica, como así se describe en los resultados de este estudio.

Entendemos que esta variabilidad existente no propicia el carácter homogéneo de una formación especializada, lo que es un requisito indispensable para lograr formar a especialistas con el nivel más alto de calidad ${ }^{(16-18)}$.

La participación de los profesionales de enfermería en las subcomisiones de docencia de las Unidades Docentes Multiprofesionales es algo que no se puede obviar y que resulta absolutamente necesario como así se justifica en numerosos estudios ${ }^{(16-19)}$. Aún hay muchas presidencias de las subcomisiones de docencia que no están ocupadas por profesionales de enfermería, siendo un hecho que dificulta una formación de calidad para los futuros especialistas, los cuales servirán de guía en un nuevo modelo de Atención Primaria más eficiente, donde se visibilice a los profesionales de Enfermería Familiar y Comunitaria en el lugar que les corresponde $(10,20,21)$

El tutor es el primer responsable del proceso de enseñanza-aprendizaje del residente y mantiene con este un contacto continuo y estructurado en cualquiera que sea el dispositivo de la Unidad Docente Multiprofesional en el que se forme ${ }^{(22,23)}$. Aspecto que afirma la mayoría de los encuestados y que creemos que hay que resaltarlo porque de él depende en mayor medida el éxito formativo de los nuevos especialistas en Enfermería Familiar y Comunitaria. Un dato no tan bueno es que en algunos casos la elevada carga asistencial de la consulta del tutor dificulta la docencia con el residente, situación que podría justificarse con la baja dotación que existe de profesionales de enfermería con respecto a medicina en el estado Español constituyendo un desequilibrio estructural endémico ${ }^{(20,24)}$.

Las Sociedades Científicas de Enfermería Familiar y Comunitaria son conocidas por la gran mayoría de los encuestados, pero solamente la mitad de ellos considera que están impulsando y trabajando por el desarrollo de la especialidad de Enfermería 
Familiar y Comunitaria. Semeja un dato contradictorio tras la activa participación que tuvieron todas estas Sociedades Científicas en la elaboración del programa formativo de la especialidad ${ }^{(2,6,7)}$, así como también su participación en la Comisión Nacional de la especialidad ${ }^{(4)}$ y la organización de encuentros nacionales para los residente ${ }^{(15,16) \text {. }}$

Actualmente muy pocos especialistas en Enfermería Familiar y Comunitaria se encuentran desarrollando su actividad profesional en el ámbito de la Atención Primaria. Para poder aumentar este porcentaje y dadas las características organizativas del Sistema Nacional de Salud, compete a los diferentes servicios de salud de las Comunidades Autónomas regular los procedimientos de selección temporal y vinculación fija para garantizar que en el ámbito de la Atención Primaria trabajen estos profesionales. Modificaciones legislativas que fueron ya realizadas en las Comunidades Autónomas de Valencia (25), Asturias (26), Castilla y León (27) y Castilla la Mancha ${ }^{(28)}$. Pero a pesar de que hay comunidades que se encuentran negociando el proceso de modificación normativa, todavía se puede decir que la especialidad de Enfermería Familiar y Comunitaria no se encuentra implantada en la totalidad del estado Español ${ }^{(12,21)}$. El Ministerio de Sanidad ha publicado en el mes de Mayo de 2014 una propuesta de cronograma para la creación, implantación y dotación de plazas de las categorías de enfermería especialista en los distintos servicios de salud en la que se fija el año 2017 como límite para la creación de la categoría profesional de Enfermero especialista en Enfermería Familiar y Comunitaria en los distintos servicio de salud del Estado Español ${ }^{(29)}$.

Las Organizaciones Sindicales como organismos de representación de la clase trabajadora son una pieza fundamental para la consecución de este cambio normativo para facilitar la entrada al mercado laboral de los especialistas. Pero aquí existen múltiples intereses que están dificultando las modificaciones normativas necesarias, aunque ya algunas Organización Sindicales han firmado diversos acuerdos y comunicados para lograra la inserción laboral de estos profesionales de enfermería ${ }^{(30)}$.

Son destacadas las opiniones que relatan algunos de estos profesionales de enfermería cuando se les pregunta sobre qué aporta a la Atención Primaria un especialista en Enfermería Familiar y Comunitaria. Todas las opiniones coinciden en el análisis de una visión del cambio en el patrón epidemiológico de la población que está produciendo un aumento de los procesos crónicos y dependientes cada vez más costoso, lo que obliga al sistema sanitario a adoptar estrategias que pasan por procesos que sean atendidos por profesionales que garanticen la mejor eficiencia del sistema sanitario. En el contexto de la Atención Primaria la Enfermería especialista en Atención Familiar y Comunitaria tiene la necesidad y la tarea de reconducir un modelo en el que el autocuidado en la prevención, promoción y seguimiento de la enfermedad sea el pilar básico de sus intervenciones ${ }^{(9,11,12,20)}$.

Por lo que resulta de vital importancia ofrecer una formación especializada en Enfermería Familiar y Comunitaria lo más homogenizada posible, sirviendo como el instrumento necesario para garantizar la calidad de la formación en todo el sistema sanitario con unas estructuras formativas uniformes y adecuadas a la evolución de los conocimientos científicos y a las necesidades que demanda nuestra sociedad. 


\section{REFERENCIAS}

1. Galimany J, Herrera T, Girbau MR. Reflexiones sobre el futuro de la enfermería. Nursing. 2010; 28:62-4.

2. Bernardini DA. La enfermería en España: situación actual y posibles líneas estratégicas (carta al director). Enferm. Clin. 2012; 22 (5):278-282.

3. Millas J, Otegui A, Pérez S, Arzuaga MJ, Martínez JM, Insausti MJ. Consulta de atención primaria: ¿todo es del médico?. Aten. Primaria. $2011 ; 43$ (10):516-523.

4. Ortún V. La enfermería en el mundo que viene. Enferm. Clin. 2013; 23(2):45-47.

5. Orden SAS/1729/2010, de 17 de junio, por la que se aprueba y publica el programa formativo de la especialidad de Enfermería Familiar y Comunitaria. Boletín Oficial de Estado, núm. 157, de 29 de Junio de 2010.

6. Molas $M$, Brugués $A$, García $C$. Especialidad en Enfermería Familiar y Comunitaria: una realidad. Aten. Primaria. 2011; 43 (5): 220-221.

7. Oltra E. La especialidad en enfermería familiar y comunitaria. Enferm. Clin. 2009; 19 (3):105-106.

8. Real Decreto 450/2005, de 22 de abril, sobre especialidades de Enfermería. Boletín Oficial de Estado, núm. 108, de 6 de Mayo de 2005.

9. Real Decreto $639 / 2014$, de 25 de julio, por el que se regula la troncalidad, la reespecialización troncal y las áreas de capacitación específica, se establecen las normas aplicables a las pruebas anuales de acceso a plazas de formación y otros aspectos del sistema de formación sanitaria especializada en Ciencias de la Salud y se crean y modifican determinados títulos de especialista. Boletín Oficial de Estado, núm. 190, de 6 de Agosto de 2014.

10. Ley 44/2003, de 21 de noviembre, de ordenación de las profesiones sanitarias. Boletín Oficial de Estado, núm. 280, de 22 de Noviembre de 2003.

11. Tercer Encuentro Nacional de EIR de Enfermería Familiar y Comunitaria. FAECAP. Mallorca, 10 de Mayo de 2013. Mallorca: Asociación Balear de Enfermería Comunitaria; 2013.

12. Asociación Galega de Enfermaría Familiar e Comunitaria. Acuerdo sindical por el impulso de la especialidad de Enfermería Familiar y Comunitaria en Galicia. Enero del $2014.2 \quad$ Disponible en: http://www.agefec.org/wpcontent/uploads/2014/03/Acuerdo-sindical-por-el-impulso-de-la-efyc.pdf.

13. Asociación Galega de Enfermaría Familiar e Comunitaria. Comunicado por el reconocimiento de la especialidad de Enfermería Familiar y Comunitaria. Madrid; Julio del 2014. Disponible en: http://www.agefec.org/wpcontent/uploads/2014/08/comunidado_por_la_especialidad_julio.pdf

14. Primer Encuentro Nacional de Tutores y Residentes de la Especialidad de Enfermería Familiar y Comunitaria. Barcelona; 16-18 de Mayo de 2012. Barcelona: Asociación de Enfermería Comunitaria; 2012.

15.Segundo Encuentro Nacional de Especialistas en Enfermería Familiar y Comunitaria. FAECAP. Madrid, 9 de Marzo de 2012. Madrid: Federación de Asociaciones de Enfermería Comunitaria y Atención Primaria; 2012.

16. Oltra E, Rich M, Orts MI, Sánchez D, González P. Competencias de investigación en las especialidades de enfermería. Enferm. Clin. 2013; 23 (5): 225-230.

17. Orden PRE/861/2013, de 9 de mayo, por la que se establecen los requisitos de acreditación de las unidades docentes multiprofesionales para la formación de especialistas en enfermería familiar y comunitaria y en medicina familiar y comunitaria. Boletín Oficial de Estado, núm. 119, de 18 de Mayo de 2013.

18. Oltra E. Especialidades enfermeras: el día después. Rev. Adm. Sanit. 2009; 7 (2): 293-307. 
19. Orden SAS/2447/2010, de 15 de septiembre, por la que se aprueba la convocatoria de prueba selectiva 2010, para el acceso en el año 2011, a plazas de formación sanitaria especializada para graduados/diplomados en Enfermería. Boletín Oficial de Estado, núm. 230, de 22 de Septiembre de 2010.

20.Sánchez Cl. Cronicidad y complejidad: nuevos roles en enfermería. Enferm Clin. 2014; 24:79-89.

21. Díaz CM, García D, Borbón CD, Lobato $A L$, Suárez MP. La enfermera canaria ante los nuevos cambios académicos y profesionales. Enferm. Clin. 2010; 20 (4): 243-249.

22. Mompart MP. Especialidades y enfermería de práctica avanzada. Revista Iberoamericana de Enfermería Comunitaria. 2011; 4:17-21.

23. Pastor FJ. Análisis DAFO de la situación actual de la Especialidad de Enfermería Familiar y Comunitaria. EN: Libro de ponencias: 10ํㅡㄹ Congreso de la Asociación Andaluza de Enfermería Comunitaria. Torremolinos; Asociación Andaluza de Enfermería Comunitaria; 2012.

24. Guzmán MA. Pérdida de poder de las direcciones de enfermería de Atención Primaria. Enferm. Clin. 2011; 21(4):187-188.

25. Real Decreto 183/2008, de 8 de febrero, por el que se determinan y clasifican las especialidades en Ciencias de la Salud y se desarrollan aspectos del sistema de formación sanitaria especializada. Boletín Oficial de Estado, núm. 45, de 21 de Febrero de 2008.

26. González B, Barber P. Planificación y formación de profesionales sanitarios con foco en la atención primaria. Informe SESPAS 2012. Gac. Sanit. 2012; 26:46-51.

27. Decreto 70/2013, de 7 de junio, del Consell, por el que se ordenan diversas categorías de personal estatutario de la Agència Valenciana de Salut. Diari Oficial de la Comunitat Valenciana, núm. 7043, de 16 de Junio de 2013.

28. Resolución de 18 de junio de 2014, de la Dirección Gerencia del Servicio de Salud del Principado de Asturias, por la que se modifica el pacto sobre contratación de personal temporal y sobre promoción interna temporal del personal del Servicio de Salud del Principado de Asturias. Boletín Oficial del Principado de Asturias, núm. 144, de 23 de Junio del 2014.

29. Orden SAN/475/2014, de 29 de mayo, por la que se modifica el artículo 5 de la Orden SAN/398/2010, de 23 de marzo, por la que se establece el procedimiento de funcionamiento de las bolsas de empleo para la selección del personal estatutario temporal del Servicio de Salud de Castilla y León. Boletín Oficial de Castilla y León, núm. 113, de 16 de Junio de 2014.

30. Resolución de 09/04/2014, de la Dirección General de Relaciones Laborales y Seguridad y Salud Laboral, por la que se acepta el depósito y se dispone la publicación del Pacto sobre Selección del Personal Temporal del Servicio de Salud de Castilla-La Mancha. Diario Oficial de Castilla-La Mancha, núm. 82, de 2 de Mayo de 2014. 


\section{Anexo I: Cuestionario de recogida de datos.}

1. Edad (años)

2. Sexo

a. Hombre

b.Mujer

3. Categoría

a. Residente EFyC

b.Especialista EFyC

4. Promoción
a. $2011-2013$
b. $2012-2014$
c. $2013-2015$
d. $2014-2016$

5. Año actual de residencia (si procede)
a. $R-1$
b. $R-2$

6. Al elegir esta especialidad, indica el orden de preferencia que ocupaba para ti en la elección de plaza EIR.

7. De tener que escoger esta especialidad de nuevo, indica el orden de preferencia que ocuparía.

8. Valora el grado de cumplimiento de las competencias de la especialidad de EFyC recogidas en el BOE durante tú proceso de formación:

a. Cumplo con todas las competencias

b.No cumplo con todas las competencias.

9. En qué ámbito de tú programa formativo no cumples con las competencias exigidas (texto libre).

10. Has tenido o tienes alguna dificultad durante la residencia para adquirir las competencias en algún rotatorio.

a.Si (indica cuál)

b.No

11. Cómo se organiza la parte teórica de la formación en tú Unidad Docente Multiprofesional (UDM).

a.Clases teóricas más cursos de formación.

b.Cursos de formación exclusivamente.

c.Otras formas.

12. El presidente de la subcomisión de la especialidad de tú Unidad Docente es un profesional de:

a.Enfermería 

b.Medicina
c.Desconozco su categoría profesional

13. Estas satisfecho con el apoyo a la investigación que te ha proporcionado o te proporciona tú Unidad Docente Multiprofesional (UDM).

$\begin{array}{llllllll}\text { Nada satisfecho } & 1 & 2 & 3 & 4 & 5 & \text { Totalmente satisfecho }\end{array}$

14. Tú Unidad Docente para finalizar la residencia te exige:

a.Trabajo de investigación

b.Proyecto de investigación

15. La UDM os permite realizar una evaluación anual de vuestro tutor.
a.SI
b.No

16. Valora la calidad asistencial de tú tutor.
a. Excelente
b.Muy buena
c.Buena
d.Pobre
e. Regular

17. Valora la calidad docente de tú tutor.
a. Excelente
b.Muy buena
c.Buena
d.Pobre
e. Regular

18. Como registra tú actividad formativa durante el período de residencia
a.Portafolios
b.Libro del residente
c. No registro mi actividad formativa
d.Otro

19. La carga asistencial en la consulta de tú tutor de Atención Primaria facilita la docencia.
a.Si
b.No

20. A tú juicio, como cuantificarías el esfuerzo que hace la Sociedad Científica a la que perteneces 0 en su defecto las Sociedades Científicas en general para conseguir tú inserción laboral como especialista en el ámbito de la Atención Primaria.

$$
\begin{array}{lllllll}
\text { Nada } & 1 & 2 & 3 & 4 & 5 & \text { Mucho }
\end{array}
$$

21. Tienes o has tenido contacto con las Organizaciones Sindicales para exponerles la situación laboral de los especialistas en EFyC.
a. Si
b.No

22. Ordena estos temas por el orden de prioridad que tiene para ti actualmente. 
- Creación de la categoría profesional.

- Listas especiales que favorezcan el acceso de los especialistas a la AP.

- Celebración del examen para optar al título de especialista por la vía extraordinaria.

23. En tú comunidad autónoma el tiempo trabajado como residente puntúa como tiempo efectivo de servicio prestados en el baremo de la bolsa de contratación.
a.Si
b.No

24. Crees que la situación de los especialistas va a cambiar en un corto especio de tiempo (2 años).
a.Si
b.No

25. Estas desarrollando tú actividad profesional en el ámbito de la Atención Primaria (contestar solo por especialistas).
a. $\mathrm{Si}$
b. No

26. En caso afirmativo, podrías indicar que tipo de contrato tienes.
a. Eventual
b. Interino
c. Fijo

27. Poseer el título de especialista ha facilitado tú entrada al mundo laboral de la Atención Primaria.
a. Si
b. No

28. Qué puede aportar un profesional de enfermería especialista en EFyC a la Atención Primaria (texto libre).

Recibido: 13 de abril 2015; Aceptado: 24 de junio 2015

ISSN 1695-6141

(C) COPYRIGHT Servicio de Publicaciones - Universidad de Murcia 